\title{
"CONSTRUCCIÓN DE SIGNIFICADOS DE EXPERIENCIA DE CUERPO CON PERSONAS EN SITUACIÓN DE DISCAPACIDAD FÍSICA ADQUIRIDA"
}

\author{
Macarena Gálvez L ,, Marcos Godoy L²., Alejandra Lagos F ${ }^{3}$.
}

\section{Resumen:}

En el contexto de los nuevos desarrollos teóricos y metodológicos en Terapia Ocupacional, surgen perspectivas cualitativas de investigación y una compresión social de la disciplina, desde esta perspectiva, nos hemos interrogado con respecto a la significación del cuerpo y la discapacidad.

El estudio profundiza en el tema presentado, desde una óptica de los fundamentos y su expresión en la práctica de la Terapia Ocupacional (T.O.). En el análisis, observamos cómo se enuncian ideas que aluden a la unión entre lo objetivo y subjetivo, entendiendo lo objetivo como aspectos naturales, fisiológicos, anatómicos, y lo subjetivo como las ideas de unión mente-cuerpo, historia de vida y contexto sociocultural. Se propone una mirada del cuerpo como hecho subjetivo, sociohistórico, asumiendo que no tiene un punto de partida en una materialidad abstracta, separada del sujeto mismo. Al respecto, se plantea el siguiente objetivo de investigación: Explorar qué significados de experiencia de cuerpo construyen las personas en situación de discapacidad física adquirida.

El método de investigación utilizado son Relatos de Vida para construir narraciones relacionadas a la experiencia subjetiva de cuerpo. En cuanto a las técnicas, se aplicaron entrevistas en profundidad, y el análisis y producción de datos se apoyó en la Teoría fundamentada. La muestra la constituyó un grupo de personas adultas con el diagnóstico de discapacidad física adquirida.

Los principales resultados se relacionan con los puntos de inflexión que identificamos en los relatos de vida, y que a su vez son fundamentales en la construcción y significación de la experiencia de cuerpo.

\section{Palabras claves}

Cuerpo y subjetividad, Cambio paradigmático.

1 Terapeuta .Ocupacional., Magíster en Terapia Ocupacional mención Salud Física. mac.galvez@gmail.com

2 Terapeuta .Ocupacional Magíster en Terapia Ocupacional mención Salud Física. Mar_godoy@hotmail.com

3 Terapeuta .Ocupacional Magíster en Terapia Ocupacional mención Salud Física. alejandralagosfernandez@gmail.com 


\section{Summary}

In the context of new theoretical and methodological developments in Occupational Therapy, emerge new qualitative perspectives of research and a social understanding of the discipline, for this reason we have questioned regarding the signification of body and disability.

The following study depths in the mentioned topic from the point of view of basis (paradigms, approaches and models) and its expression in Occupational Therapy (OT) practice. In this analysis, we observe how some ideas are alluded in the union between the objective and subjective, defined objective as natural, physiological, anatomical aspects, among others, and the subjective as the ideas of the mind-body union, history of live, social-cultural context, among others.

The present study aims to propose a view of the body as a subjective fact, sociohistorical, assuming that it does not have a starting point in an abstract substance, separated from the subject himself. Regarding to this, it is posed the following research objective: To explore which meanings of body experience build people suffering from acquired physical disability.

The research method used was The Life Stories to build narratives related to the subjective experience of the body, In regard to the techniques, they were applied in-depth interviews, and data analysis and production was supported by the grounded theory. The sample comprised a group of adults diagnosed with acquired physical disability.

The main results are related to the turning points that we identified in the life stories, which at the same time are fundamental in the construction and significance of body experience.

\section{Keywords}

Body and subjectivity, paradigm shift.

\section{INTRODUCCIÓN - ProblematizaCIÓN}

La siguiente investigación surge a partir de nuestra experiencia inicial como Terapeutas Ocupacionales. En este breve transito en el que hemos participado en la práctica, fuimos espectadores de cómo ciertos ámbitos de intervención de la T.O muchas veces no están en sintonía con los fundamentos bases de nuestra disciplina, todo ello experienciado principalmente en el ámbito de salud física adulto. Este cuestionamiento nace debido a nuestra formación universitaria, donde se nos transmitió que la perspectiva o realidad psicosocial es inherente a cada uno de nosotros, siendo una realidad a tener presente al momento de intervenir, sin embargo, en la práctica en salud físico, vemos muchas veces como lo psicosocial se supedita ante un saber biomédico que predomina de manera histórica en esta área de intervención, interfiriendo en el planteamiento de una intervención integral, holística y en la cual debería existir, entre otros, una unión mente cuerpo de manera inextricable, tal como lo proponen nuestros propios fundamentos.

El predominio del saber biomédico en T.O -principalmente en el área de intervención en salud física- es la consecuencia de la propia historicidad de la disciplina. Durante el desarrollo del paradigma mecanicista tomamos fuertemente las raíces biomédicas predominantes de la época, capturando con ello un lenguaje y mirada médica que está aun presente en este ámbito de intervención y que apuntan a un lenguaje reduccionista, objetivo, biológico, propio de las ciencias naturales, donde la historicidad y cultura son desplazados hacia un plano subjetivo el cual no se puede develar, pues con ello se transgrede la relación vertical profesional-"paciente" que la perspectiva biomédica impone de manera tácita pero absolutamente efectiva en la práctica de la T.O. 
Todo este cuestionamiento decanta en el cuerpo. Este para lo biomédico se convierte y es su objeto predilecto de estudio, considerándolo como un cuerpo biológico, compuesto por partes, subsistemas, funcionante en base a leyes de la física y biología, por ende ahistórico, entendiéndolo abstraído de su propia experiencia personal, histórica, política y cultural, es decir, un cuerpo aislado. Lamentablemente la historia de la T.O en el ámbito de salud física toma a este cuerpo -objeto de saber- y lo hace su propio objeto de intervención, olvidando el lado subjetivo del mismo, entendiendo "subjetivo como lo resultante de la interacción entre individuos, grupos y clases, donde el sujeto no es sólo un sujeto relacionado, sino también es un sujeto producido, es decir, no hay nada en él que no implique la presencia de otro social culturizado" (1). Por esto nuestra investigación se centra en el cuerpo, y adentrarnos en qué concepto de cuerpo ha construido la terapia ocupacional y cómo este se ve impactado al adquirir una discapacidad, convirtiéndose esto para nosotros en un punto de inflexión donde se pueden valorar cambios en el cuerpo natural que la T.O tiende a abordar, pero que también se van produciendo transformaciones en la experiencia subjetiva de cuerpo/discapacidad, narrado por los propios protagonistas.

Dentro de la revisión bibliográfica que efectuamos para apoyar nuestra investigación, y dicho sea de paso es información elaborada desde las ciencias humanistas, encontramos autores que representan lo que sucede en la T.O. Estos incorporan ideas que aluden a lo objetivo (cuerpo material/cuerpo carne) y lo subjetivo (cuerpo sujeto histórico y culturizado), sumado al punto de inflexión de adquirir una discapacidad. De este modo Ferrante y Ferreira (2007) refieren que "(...) de este modo la discapacidad poseería dos dimensiones: existe una "situación de discapacidad", es decir, ciertas condiciones generales - tanto cuantitativas como cualitativas- que la discapacidad incorpora como atributos objetivos en un contexto en particular, y una "condición de discapacidad", que evidenciaría la experiencia individual de esas condiciones generales objetivas como vivencia subjetiva de la persona. Así situación y condición han de ser en- tendida como dos caras o aspectos inseparables de la discapacidad: la cara "objetiva" y la cara "subjetiva" de la misma" (2). De acuerdo a esto nosotros apoyamos que tanto cuerpo y discapacidad son caras inseparables, y de hecho esta idea es la que apoya y está en sintonía con uno de los fundamentos teóricos discutidos durante la investigación: unión mente cuerpo.

Nuestro sentir no es criticar la práctica de la T.O que opera bajo un enfoque mecanicista, por el contrario, lo que pretendemos es - a partir de la propia narración de los actores - mostrar la importancia de reconocer la existencia de un cuerpo subjetivo que al experienciar una discapacidad no sólo se transforma en términos físicos u objetivos, sino también en términos subjetivos.

La relevancia teórica es contribuir a un cambio de abordaje paradigmático en la disciplina, que incorpore nuevos elementos desarrollados por otras disciplinas humanistas, produciendo un punto de encuentro, que contribuyan a un abordaje psicosocial y subjetivo del cuerpo y discapacidad. Con ello pretendemos promover la discusión en torno al concepto de cuerpo, y poner en juego la mirada biológica y mecanisista que la terapia ocupacional ha establecido en relación al cuerpo, versus la valoración subjetiva del mismo. La relevancia práctica se centra principalmente en aportar a la posibilidad de contribuir a la transformación práctica de la T.O, introduciendo una nueva mirada del sujeto, favoreciendo la necesidad de concebir las experiencias personales y corporales. Además a partir de los resultados se pueden constituir ideas relacionadas con los significados de cuerpo que pueden transformarse en una nueva forma de abordaje terapéutico. Finalmente la relevancia metodológica es la utilización de relatos de vida para explorar el significado de la experiencia de cuerpo de personas en situación de discapacidad.

\section{Marco MetodolóGico.}

El diseño de investigación es cualitativo donde se aborda al sujeto como un hecho histórico y social, interpretando el discurso de los diferentes actores socia- 
les de manera particular y colectiva situado desde un contexto social, político, cultural y subjetivo. El conocimiento se produce y construye en conjunto con los actores sociales ya que la investigación pretende explorar significados de experiencias de cuerpo construidos con las personas en situación de discapacidad física adquirida. Considerando las características de la realidad estudiada, requerimos utilizar un método que sea flexible, abierto y progresivo.

Utilizamos Relatos de Vida para construir narraciones en relación a la experiencia de cuerpo. Éstos pertenecen al Método Biográfico, definido por Ruth Sautu como "conjunto de técnicas metodológicas basadas en la indagación no estructurada sobre las historias de vida tal como son relatadas por los propios sujetos" (3). También para esta autora adquiere importancia no sólo la biografía de las personas, sino también, los aspectos políticos, económicos y de organización social de los sistemas en los que viven, además del comportamiento interpersonal y los mecanismos psicológicos y cognoscitivos de los individuos.

Chirico M, (1992), plantea que los relatos de vida se caracterizan por tres elementos que sustentan su uso:

- La existencia de un "yo" que es protagonista de los contenidos, sucesos o procesos analizados en el estudio.

- Esos sucesos o procesos tienen lugar en contextos historico-políticos y sociales de diversos tipos (familiares, amigos, de trabajo, etc.).

- Existen puntos de inflexión que señalan la presencia de cambio o marcan aspectos destacables del transcurso de vida (4).

Para la obtención de la información que permita la construcción de los Relatos de Vida, realizamos Entrevistas en Profundidad, centrando las narraciones en el tema a investigar, es decir, qué significados de cuerpo construye el sujeto al experienciar la adquisición de una discapacidad. Las entrevistas no fueron realizadas a través de preguntas dirigidas a través de un guión, sólo previamente definimos ejes temáticos esenciales para abordar la construcción de la experiencia de cuerpo. Los ejes temáticos previos que definimos fueron temas abiertos en torno al quién soy, con el objeto de conocer cómo la persona se reconoce e identifica a sí misma. El segundo tema se ideó con el objeto de que la persona libremente expresara y contara su historia personal en torno a la vivencia de discapacidad, y finalmente el tercer tema alude al cuerpo, en relación a las experiencias naturales y subjetivas del mismo.

La ideación de estos ejes temáticos apuntan a relacionar la construcción de cuerpo como un proceso dinámico y en continuo desarrollo, los cuales se encuentran a su vez en estrecha relación con las historias de vida de los sujetos situadas en un contexto histórico, político, económico, social y cultural, a partir del cual el sujeto resignifica y construye constantemente su experiencia de cuerpo. En este continuo propuesto consideramos la adquisición de una discapacidad como un dispositivo que produzca un cambio en dicha construcción.

\section{Construcción y justificación de la muestra.}

La selección de la muestra se hizo de forma intencionada para obtener discursos de sujetos que ocupan un lugar social específico, relacionado con nuestro tema de estudio. Además a priori establecimos criterios muéstrales para obtener mayor variabilidad discursiva, los cuales fueron: la adquisición una discapacidad física -como dispositivo de cambio- de carácter transitoria o permanente, y el género.

Entonces la adquisición de una discapacidad actúa como un dispositivo significativo que fractura en términos simbólicos la construcción de experiencia de cuerpo, lo que nos permite valorar cambios no sólo en la condición natural del cuerpo, sino también visualizar el cambio social, histórico y cultural del sujeto y su entorno a partir de esta experiencia. Además, realizamos la construcción focalizada en la discapacidad física, pues en este tipo de discapacidad la T.O. expresa su práctica en el ámbito de intervención en salud física. Este dispositivo tiene dos formas de adquisición: permanente o transitoria. 
Además de los criterios antes mencionados optamos por incorporar el género como criterio muestral, ya que escogimos una perspectiva social, histórica y cultural de la experiencia de cuerpo, por lo tanto asumimos la diferencia existente en los discursos entre hombres y mujeres, debido a que el ser hombre o mujer también en es una construcción social y cultural.

Por medio de la entrevista, se conocen aspectos personales de la vida de las personas entrevistadas, por lo cual se asegura confidencialidad de los antecedentes personales, donde se destaca que la información que emerge a través del relato será utilizada con fines académicos para la investigación.

El análisis de la información se realiza utilizando algunos elementos de la teoría fundamentada. Esta teoría fue originalmente desarrollada por Glaser y Strauss (1967) y surge como alternativa a las teorías dominantes de carácter funcionalista y estructuralista (5). "Esta teoría no constituye un método o una técnica específica, sino que más bien un estilo de "comparaciones constantes". Esta teoría puede aplicarse a diversos problemas siempre y cuando sean de tipo social y cualitativo (3).

Este enfoque propone dos estrategias para desarrollar una teoría fundamentada, la primera es el método de comparación constante. En éste, el investigador "simultáneamente codifica y analiza los datos que le permitirán desarrollar ideas teóricas" (3). La segunda, es la técnica del muestreo teórico, "mediante la que el investigador selecciona nuevos casos a estudiar según su potencial para refinar y/o expandir los conceptos y teorías ya desarrolladas." (3). Para efectos de la investigación utilizamos la primera estrategia del método de comparación constante.

Si bien la teoría fundamentada propone generar teorías o modelos a partir de los datos, no es el fin de nuestro estudio, ya que es un estudio principalmente de carácter exploratorio y emergente. Sin embargo, utilizamos las técnicas de análisis de datos con el objeto de establecer lineamientos y ejes bases para la realización de futuras investigaciones, que idealmente se transformen en teorías.

\section{CuerPo/trauma/CONSTRUCCIÓN EN LO} SOCIAL: ANÁLISIS DE LOS DATOS.

Hablamos de construcción en lo social, debido a que creemos que las narraciones han sido producidas de manera conjunta, a través de la dinámica de la conversación y donde los relatos van adquiriendo consistencia y significado.

A partir de estos relatos construimos dos ejes trascendentales que estructuran el análisis, los cuales fueron determinados una vez realizada la construcción de relatos de vida y posterior al primer análisis de la información. Es importante destacar que para nosotros es sumamente complejo generar un discurso que transmita la unicidad entre mente y cuerpo, o entre objetivo y subjetivo, pues nosotros mismos hemos sido configurados desde esta dicotomía en el lenguaje, generada o preformada en nuestra propia cultura occidental o en nuestro propio mundo académico o formativo como terapeutas ocupacionales.

El primer eje lo denominamos Cuerpo Traumatizado, entendiendo como trauma a un cambio vivenciado no sólo en la dimensión física o material, sino que también es comprendido como un cambio en términos subjetivos emocionales y sociales que se relaciona con la adquisición de la discapacidad. El segundo eje se denomina El cuerpo en la construcción social, donde se observa cómo los sujetos construyen significados de experiencia de cuerpo en relación con su entorno, como un hecho social producido en el espacio social e intersubjetivo.

\section{Cuerpo Traumatizado:}

Hacemos referencia a este eje para unificar aquellos relatos que se construyen en estrecha relación con la experiencia del trauma y la adquisición de la discapacidad, que consecuentemente expresan de manera implícita en las narraciones ciertos cambios en diversas dimensiones en la experiencia de vida de las personas. La primera pregunta, marca claramente como el trauma 
se transforma en un claro elemento que estructura el discurso:

"E: Nos gustaría que nos contara ¿Quién es usted? ¿En general o desde que me enfermé?"

El extracto anterior ejemplifica cómo a raíz del trauma la persona organiza su significación personal en torno a esta vivencia, haciendo un quiebre en su historia de vida y marcando de esta forma un antes y un después. Consecuentemente, podemos ver que estas narraciones apoyan uno de nuestros supuestos -la adquisición de una discapacidad física actúa como dispositivo que produce un quiebre en la construcción de la experiencia de cuerpo-. Esta experiencia de cuerpo, como anteriormente mencionamos, incorpora una visión del cuerpo en términos naturales y subjetivos, o desde el hacer y ser un cuerpo, considerándolo como elemento importante en ésta construcción de significación personal.

$\mathrm{Al}$ adquirir una discapacidad se inicia un proceso de darse cuenta, de reconocerse a sí mismo en términos naturales y subjetivos, es decir, es aprehender a conocer un cuerpo nuevo. En las diferentes narraciones podemos apreciar que los actores expresan un darse cuenta de lo que pasa con el cuerpo en términos naturales, de cómo la persona vive ese momento. Vemos cómo a medida que las sensaciones corporales sentidas en el accidente se van haciendo conscientes actúan como un movilizador de que el cuerpo está cambiando y es enfrentarse a la posibilidad real de dejar de moverse y a todo lo que esto implica: la adquisición de una discapacidad. Es un sentir diferente que es imposible no compararlo con lo establecido, es dejar de sentirse normal.

Otro punto importante de mencionar emerge de los relatos pertenecientes a mujeres, sin importar el tipo de discapacidad física que adquieran, en ellas la estética emerge como un elemento distintivo y que influye en la construcción de cuerpo. Esta vinculación a lo estético tiene un origen social y cultural en relación a lo que la sociedad ha estipulado como normal en torno a lo bello, dejando entrever en los relatos que la narración nunca deja de relacionarse estrechamente con el cuerpo legítimo-bello-normal-funcional, concepto predefinido culturalmente, y consecuentemente los sujetos vivencian su propio cuerpo como un cuerpo ilegitimono bello-anormal-disfuncional. (6).

Un elemento que emerge del análisis de los relatos, dice relación con lo que nosotros denominamos el cuerpo como aprendiz, puesto que entendemos este concepto como aquellos nuevos conocimientos prácticos que surgen a través del cuerpo, a raíz de la experiencia de discapacidad en términos del hacer cotidiano. Ella hace referencia a un cuerpo que no responde y frente a esto, el cuerpo se adapta, busca estrategias y aprende nuevas formas de hacer de manera consciente.

“(...) yo me emocionaba por las cosas nuevas que hacía, me enseñaron con velcro aquí en la mano a comer, pero llegue a la casa y después quise agarrar la cuchara y me la metí aquí, y empecé a comer al tiro en la casa (...) después llegue a la casa, mi papá me llevó una lapicera con goma, un bic cualquiera, la puse aquí y escribí igual con la misma letra, hice mi firma y todo fue súper emocionante".

El cuerpo se siente diferente, toda experiencia es nueva, por lo tanto, el enfrentarse a la actividad es percibido como un desafío que implica aprender y adaptarse, no sólo en términos físicos, si no también es reconocer el cuerpo y la actividad. Este cuerpo como aprendiz se refleja en el hacer cotidiano, pero a la vez en los relatos encontramos elementos que van más allá del aprendizaje, es decir, cuando los actores logran hacer suyos estos aprendizajes. Este proceso para fines del análisis lo denominamos Aprehender a conocer el cuerpo. Éste se refiere a cómo las personas a partir de la experiencia de discapacidad aprehenden a comprender, escuchar y conectarse con su cuerpo, con un cuerpo vivido por ellos mismos como diferente, y de esta forma aprehenden a entender a un nuevo cuerpo y a ser con él, haciendo referencia a un proceso de autoconocimiento en constante construcción. 
"E: Y... haber, ésta es una duda personal que yo tengo, esta relación que tú tienes con tu cuerpo que decía $M$, ¿tú crees que es diferente hoy 10 años después en relación a como fue tu primer año después del accidente?

Al principio claro desconoces tu cuerpo, totalmente desconoces tu cuerpo, no sabes que... uno ahora lo empieza a conocer, a detectar ciertas cosas que al principio uno no tiene idea, ciertos estímulos, o sea, eso a cambiado... ahora yo me manejo muy bien con mi cuerpo, lo conozco..."

En relación a las ideas anteriormente planteadas, el cuerpo como aprendiz y aprehender a conocer el cuerpo, devela la importancia de valorar las vivencias personales y las construcciones en relación al cuerpo que puedan emerger desde ellas en relación a lo cotidiano, pues su experiencia permite el desarrollo de un "saber práctico", que desde el punto de vista de Bourdieu (1991) "no es reductible al conocimiento teórico y que sólo puede ser captado a través de los agentes, o más precisamente, mediante la observación de los cuerpos en movimiento y en relación con los otros" (6).

Uno de los temas trascendentes y transversales en todas las narraciones, y de carácter fundamental para nuestra investigación, es lo que denominamos Cuerpo disociado. Lo entendemos como el proceso en que las personas con discapacidad tienden a valorar a su cuerpo, o la parte afectada como algo externo a ellos.

"E: En relación a lo que tú sientes, las sensaciones, emociones, experiencias, ¿hay algo más que quieras compartir sobre eso con nosotros?

A ver, al principio me llenaba la sensación de sentir un orgasmo, a veces sentía que estaba mi miembro erecto, pero él po cachay, no tenía esa conexión, entonces era como raro, a parte era cuando él quería, o sea, yo he tenido penetración con mi pareja y todo, lo que más añoraba antes era poder sentir un poquito ese placer, esa sensación yyy... y después no sé, sin querer me di cuenta que habían otras cosas que me mataban, que me tocaran algunas partes me remecía, o sea, pa qué me voy a poner hostigoso con la cuestión si ya sé que, que aunque quisiera mentalmente provocar una, no voy a poder (...) creo que ya se me ha pasado este problema que yo tenía, que sentía que mi pene estaba erecto y que no, ¿y yo?, me cachay como que estábamos...

E: ¿Disociados?

Claro, él cuando quiere y yo no sé jajaja, es como me tomo un viagra solo".

En todas las construcciones se dota al cuerpo natural con una visión de exterioridad, valorando al cuerpo de manera distanciada del sujeto, estableciéndose una relación particular de la persona con el segmento corporal dañado. Esta relación evidencia una disociación mente y cuerpo dada por el quiebre en la construcción de las experiencias de cuerpo. Entonces mente y cuerpo, no son vivenciados como elementos inextricables, sino que el cuerpo se vive como liberado de la mente, es decir, no tiene control, se expresa cómo quiere y cuándo quiere, por ejemplo, a través de espasmos, o erecciones involuntarias.

Nos parece importante este concepto que se ha evidenciado en las narraciones construidas que muestra un quiebre en la construcción de la experiencia de cuerpo, y una indudable disociación del cuerpo en términos del ser y del hacer, es decir, de lo subjetivo y de lo natural. Esto se vincula con los supuestos teóricos de nuestra investigación, en los cuales se alude a una constante separación en la práctica de la T.O. entre la mente y el cuerpo. Esto hace relevante que la separación se haya evidenciado en los relatos, ya que reafirma la importancia de abordar al cuerpo como uno solo, construido no sólo en términos naturales, sino que es un proceso en continua construcción, constituido en el espacio social e histórico.

Al leer los relatos, emerge en nosotros el cuestionamiento de la importancia que pueden llegar a tener nuestra práctica disciplinar, y también de otras disciplinas en la construcción de cuerpo, no sólo natural, si 
no que también subjetivo, lo que ejemplificamos en la siguiente narración:

"E: ¿Y el proceso de rehabilitación, cómo fue, cómo lo viviste? ¿Cómo viviste la parte social de la " $U$ "?, ¿Que la mano te la mirara cualquier persona en el metro o la órtesis?

Es que en la "U" fue todo mejor, en el metro no, pero la órtesis sí, fue cuático, con un amigo me fui a hacer la órtesis y con otra amiga la fui a buscar y pa ponérmela el primer día... era escandalosa, y yo decía que no quería ponérmela y mi amiga me obligó.

E: ¿Por qué no querías ponértela?

Porque llamaba demasiado la atención.

E: ¿Tú encuentras que evidenciabas la discapacidad usando la órtesis?

Se aumentaba, me sentía más discapacitado usando la órtesis, si me la evidenciaba socialmente".

Este elemento es transversal en todos los relatos, donde se hace referencia al cuerpo vivenciado como un objeto de intervención; decimos objeto porque las propias personas perciben a su cuerpo como receptor y prisionero de intervención, ya que habitualmente las prácticas profesionales se centran en aspectos concretos y materiales a mejorar, focalizando la intervención en la enfermedad y disfunción por sobre la propia persona, siendo de este modo la persona un ser pasivo dentro de todo el proceso de tratamiento y rehabilitación.

\section{Cuerpo en la Construcción Social}

Este segundo incorpora experiencias relacionadas con el cuerpo y su interacción con el entorno social o físico. Todos los relatos tienen fuertes directrices que marcan la interacción del cuerpo con lo social. Estas experiencias las hemos agrupado bajo el nombre del Cuerpo en lo social.
"E: ¿Qué es lo que más te afectaba en ese momento, más que el tema del cáncer que ya nos contaste que fue heavy para ti, más por el tema motor?

Lo que más me afectaba era no poder hacer lo mismo que mis compañeras hacían, porque cuarto medio igual es como la época en que uno lo pasa mejor en el colegio, está lleno de celebraciones, de instancias importantes como de reflexión y que no las pude vivir, por el hecho de no ir al colegio $y$ de no poder asistir a todos estos momentos importantes de curso, que fue mi curso de prekínder a cuarto medio, entonces amigas de toda la vida, eso fue lo peor, como que mi limitación motora y biológica, pero que motora antes que biológica, no me permitirán compartir con las personas que quería y en los lugares que yo quería, porque mis compañeras iban mucho a la casa, hacíamos muchas oncecitas y cosas sanas, pero en realidad lo que todas queríamos, o sea, lo que yo quería era estar en fiestas como ellas, salir, estar en la onda de los 18 años".

En el relato ejemplificamos cómo la edad o ciclo vital se convierte en un punto de inflexión en la construcción de experiencias de cuerpo. Para cada ciclo vital existe un espacio social que lo identifica, se expresa como las personas están configuradas socialmente en relación a qué cosas deberían hacer y en qué espacio deberían desempeñarse en una situación o experiencia de vida normal, sin embargo, en el relato se comunica que se ha vivido una experiencia contraria a lo socialmente esperado, viviéndose como un sujeto socialmente diferente y coartado por la experiencia de la discapacidad, como un cuerpo ilegítimo. De este análisis y otros elementos emerge otro punto vinculado a lo social es la experiencia de discriminación que de manera transversal se hace presente en los relatos, y es lo que ahondaremos a continuación.

“(...) la gente hace 7, 8 años, no estaba acostumbrada a ver a tanta gente pelada en la calle, como que mi sufrimiento fue más por eso, ponte tú, una 
vez en el supermercado una señora que estaba con un niño chico, la abuela le dice "si tú no te comes la comida vas a quedar asi", entonces eso lo encontré como lo máximo, lo más hiriente que te podían decir, sino pasa por comerte o no la comida, como que fuera un castigo divino me entendís"

Es así como volvemos a reafirmar que la construcción de cuerpo se produce en el espacio histórico social donde son configurados, simultáneamente, la persona dueña de un cuerpo traumatizado y el grupo social que históricamente, a través de la cultura occidental, ha sido educado en valorar todo en relación a una norma socialmente aceptada y establecida como normal, no generando espacio o cabida a la diversidad. Vemos cómo la persona vivencia una situación de dominación a partir de esta experiencia descalificante, donde se aprecia que ser y tener un cuerpo discapacitado no tiene lugar en el campo social occidental en el que estamos inmersos, por tanto estos cuerpos socialmente son configurados desde la exclusión.

$\mathrm{Si}$ esto es lo que sucede cuando la persona pone en acción este nuevo cuerpo en un campo social amplio, ¿qué es lo que sucede cuando este nuevo cuerpo se pone en acción en su entorno inmediato, por ejemplo, a nivel familiar? En relación a esto identificamos dos aspectos que se presentan de manera global en todos los relatos, los que hemos Ilamado Reencuentro y Pilares de apoyo.

Nosotros entendemos el reencuentro como la movilización de la red de apoyo primaria que se produce en torno a la persona que ha vivido la adquisición de la discapacidad. En todas las narraciones aparece como factor transversal una red de apoyo que se activa o fortalece a causa de la experiencia, ya que es transformadora de la historia familiar y del entorno social. Los Pilares de apoyo los entendemos como los recursos en que las personas que han adquirido la discapacidad se sustentan frente a esta situación de adversidad. En éste, apreciamos cómo la familia se organiza en torno a la persona con discapacidad, entregándole un apoyo efectivo emocional y práctico. Hacemos mención a este apoyo práctico, debido a que las limitaciones funcionales, transversales en todos los relatos, producen un cambio en cuanto al nivel de independencia de la persona, traduciéndose finalmente en lo que Ilamaremos Dependencia en el hacer, comprendiéndola como la necesidad de apoyo sentida por las propias personas que han adquirido una discapacidad para efectuar ciertas actividades cotidianas.

Entonces a través del reencuentro, pilares de apoyo y dependencia en el hacer vemos cómo se despliega el capital social en torno a la persona con discapacidad física adquirida. El capital social de manera transversal en las entrevistas apoya el proceso de experienciar una discapacidad, construyendo de esta forma una trayectoria social ascendente, pues el apoyo es entregado desde el punto de vista emocional y necesidades cotidianas. También el capital social aporta el capital económico necesario para el proceso de rehabilitación y para la construcción de una trayectoria social también ascendente. La suma de capitales que tenga a disposición la persona con discapacidad hace que varíe la forma en que experiencia la discapacidad en relación a la construcción de trayectorias sociales ascendentes o descendentes (2)

\section{DisCuSiOneS}

Mediante el análisis de relatos de vida a través de entrevistas en profundidad, hemos realizado la construcción de significados de experiencias de cuerpo con personas en situación de discapacidad física adquirida con el objeto de dar respuesta a nuestra pregunta de investigación, ¿Qué significados de experiencia de cuerpo construyen las personas en situación de discapacidad física adquirida? De manera inicial nos hemos planteado dicha pregunta con el fin de contribuir a la posibilidad de proponer una postura teórica diferente, además de promover un cambio paradigmático en términos epistemológicos, en el cual no sólo exista un cambio en el cómo se valora al sujeto, sino que también se genere un cambio en el cómo nos posiciona- 
mos como disciplina en relación al sujeto o realidad en estudio. Nosotros proponemos como disciplina lograr situarnos desde un paradigma constructivista, el cual epistemológicamente es de carácter subjetivista, donde no existe una diferenciación o distanciamiento de sujeto o realidad estudiada.

La postura existente hoy, como mencionamos anteriormente, presenta una valoración naturalista del cuerpo que se expresa principalmente en el ámbito de intervención de salud física. A su vez, esta mirada sustenta dicha práctica donde se aprecia al cuerpo como un cuerpo máquina, abstracto y ahistórico de su propia experiencia. Frente a este escenario nosotros nos cuestionamos si ies posible plantearse que pueda existir y conformarse una postura diferente al respecto, que tienda a valorar al cuerpo no como una máquina que hay que reparar para que continúe funcionando, si no que valore al cuerpo como el sujeto mismo?

A partir de la experiencia que hemos vivido desde el momento en que planteamos esta investigación y durante su desarrollo, creemos con más fuerza que este cuestionamiento sí es posible debido a las implicancias prácticas y teóricas que significa el cambio de la valoración actual del sujeto. Este cambio conlleva que el cuerpo puede ser experienciado de manera diferente, como un cuerpo libre, como un cuerpo sujeto construido social e históricamente, como un cuerpo narrado por sus propios protagonistas, alejándose del lenguaje biomédico que históricamente ha predominado en torno a la temática del cuerpo y la discapacidad.

Desde el inicio, planteamos que la construcción de significados de experiencias de cuerpo es un proceso dinámico, un continium que está en constante transformación, proceso que se vincula estrechamente con las experiencias de vida, que desde la teoría de Bourdieu se denominan trayectorias sociales, refiriendo que al poseer o adquirir un cuerpo no legitimo/anormal/no bello las trayectorias sociales se modifican e interactúan con los capitales simbólico o global, social, económico y cultural. De este modo la propia historia del "agente" es configurada socialmente construyendo una trayectoria social ascendente o descendente (2).
A partir de esta postura subjetiva y social valoramos la adquisición de una discapacidad física como un dispositivo que permitiese evidenciar cómo se produce un quiebre en este ciclo de continua construcción, con el objeto de conocer qué significados de la experiencia de cuerpo han construido personas que han adquirido una discapacidad física. Sin embargo, a lo largo del análisis de los relatos pareciera que centramos el análisis en la discapacidad, esto a raíz de que son los propios actores los que ligan y asocian continuamente la construcción de significados de cuerpo a la adquisición de la discapacidad. Los propios actores lo vivencian como un ates y un después, vemos que en las narraciones evocan el evento traumático como movilizador de nuevas experiencias, de cambios y como configurador de un nuevo cuerpo, idea que se expresa claramente en el eje de Cuerpo Traumatizado.

Según los relatos, con el tiempo, la experiencia de habitar un cuerpo distinto se va haciendo conocida y propia, estableciéndose un profundo conocer del cuerpo, que según la propuesta de Bourdieu se relaciona con el saber práctico que sólo radica en el agente o protagonista de la propia experiencia. He ahí la importancia de valorar y dimensionar el rescate de la propia voz de los protagonistas para conocer este saber práctico que muchos como profesionales transmitimos en nuestra práctica, desconociendo que ese conocimiento proviene de los propios sujetos intervenidos. (6).

Entonces, el adquirir una discapacidad implica un aprendizaje del cuerpo en términos naturales, pero también en términos históricos y sociales. El término natural, se relaciona con la idea de entrenamiento, de potenciar habilidades, de aprender y re aprender a desempeñar actividades cotidianas, lo que según Foucault se relaciona con una práctica disciplinar que tiende a dominar al sujeto que está siendo entrenado, con el objeto de buscar su máximo desempeño o potencial, idea que se visualiza en la práctica interventiva en el área de salud física de la T.O. (7), lo que implica que la intervención se desarrolle en un cuerpo físico, regido por las leyes de las ciencias naturales como la física y la mecánica; y el término histórico y social alude a que 
el cuerpo intervenido es de naturaleza social, producido a través del discurso y el lenguaje, así la persona es un sujeto producido en el campo social donde dichos discursos tienen lugar y significados, es decir, el cuerpo es construido socialmente. Eventualmente el hecho de someterse a un tratamiento de manera tan acelerada interfiere en este proceso de autoconocimiento al adquirir una discapacidad, o simplemente la intervención se centra tanto en recuperar las funciones corporales que se despersonaliza la intervención, transformándose en un proceso de intervención ahistórico, situación que se presenta habitualmente en la intervención práctica de la T.O en Salud Física.

Del análisis del aprendizaje como punto de inflexión, surge un elemento fundamental a en las implicancias prácticas de la investigación, que dice relación con rescatar el propio saber práctico que deviene de la experiencia de los propios actores, es decir, no imponer técnicas de aprendizaje o formas de hacer las cosas según protocolos o teoría, sino que debemos escuchar y aprender a reconocer este saber que proviene de la experiencia y que se expresa a través de los discursos. Esta mirada enriquece la práctica de T.O y el vínculo terapéutico al validar y reconocer el saber práctico del otro, permitiendo conocer con mayor aproximación cómo el sujeto aprehende a ser y hacer con su cuerpo.

En relación al cuerpo como construcción social, emerge un elemento fundamental, la discriminación, que si bien es un tema manoseado cuando se habla de discapacidad, éste toma relevancia para nuestra investigación y se constituye en un elemento trascendental, debido a que alude a cómo el entorno social me percibe y se aproxima a mí como alguien diferente, siendo muchas veces excluido socialmente. Así como en las trayectorias sociales los sujetos experimentan situaciones de discapacidad, en sus relatos también existen fragmentos donde discriminan a otros sujetos que se ubican dentro y fuera del campo de la discapacidad, pero ¿Por qué los sujetos que experiencian una situación de discapacidad tienden a discriminar a otros, si ellos también han sido discriminados? Esta pregunta tiene su respuesta en lo social, pues las propias perso- nas en situación de discapacidad han experienciado la violencia simbólica cotidiana, generando en ellos mismos respuestas que tienden a reproducir la situación objetiva que los condena a ellos mismos (6).

Un punto de inflexión que emerge espontáneamente en la construcción de los relatos dice relación con el género. Éste vinculado con lo estética, ambas mujeres aluden este tema, transformándose en un elemento importante en la construcción de significados de experiencia de cuerpo. La estética si bien es un factor que se aprecia visualmente en el cuerpo natural, es la cultura y el contexto social el que lo produce y lo hace relevante. Según la mirada de Butler hay discursos y prácticas instaladas repetidamente en el escenario social cotidiano que tiene el efecto de que lo nombrado se hace efectivo, se materializa. Respecto a esto, el discurso y la práctica en torno a lo estético está instalado en el escenario social y lo hace perteneciente principalmente a lo femenino, siendo las mujeres performadas en torno a lo estético, llegando a hacerse obvio (8). A la vez, para Bourdieu el cuerpo bello, es un concepto que emerge en el campo social, construido por la cultura, la política e inclusive la economía, y es entendido por él como un cuerpo legítimo, es decir, lo comprende como patrones preconcebidos que crean esquemas de percepción que son interiorizados, y que no son neutros, jerarquizando de este modo preferencias y valoraciones (6).

Otro punto de inflexión que surge de las narraciones es el ciclo vital en que la persona se encuentra al momento de vivir la experiencia de discapacidad. Como mencionamos anteriormente el ciclo vital configura determinados espacios sociales y actividades propuestas y aceptadas socialmente para cada edad. De este modo cuando se adquiere una discapacidad la persona no tiene lugar en esta configuración, y consecuentemente se interfiere el desempeño en dichos espacios sociales y actividades. A raíz de esta experiencia la persona se vive a sí misma como diferente y consecuentemente se margina de participar en determinados espacios sociales, o simplemente busca nuevas estrategias o formas de relacionarse para integrarse a dichos espacios; no sólo la persona es la que se debe adaptar a raíz de la 
adquisición de una discapacidad, también el entorno debiese hacerlo. Entonces nos preguntamos ¿qué ocurre cuando el contexto no se adapta a la diversidad? Frente a esta pregunta nos apoyamos en lo que Bourdieu (1999) denomina violencia simbólica, debido a que ésta da cuenta de los procesos de exclusión social, pues a través de procesos de enfermedad o discapacidad, se naturaliza la dominación que históricamente ha predominado en el campo de la normalidad por sobre el de la anormalidad, es decir, en este caso el campo de la discapacidad. (6).

Un elemento trascendental es cómo las personas vivencian su cuerpo como un cuerpo disociado. Éste a nuestro parecer tiene su origen en la validación que el colectivo social históricamente ha tenido en relación a las ciencias médicas. La aceptación del colectivo, implica que hace suyo el entendimiento que tienen las ciencias respecto al hombre, que es considerado como un hombre máquina; de esta forma las personas reproducen sus discursos y relatos basados en esta idea.

Es así, como también las prácticas biomédicas tradicionales, han configurado al cuerpo natural-máquina, como el lugar de su intervención, obviando u omitiendo el impacto de dichas prácticas en los pacientes en términos históricos y sociales. En las narraciones los propios actores aluden a un cuerpo vivenciado como sometido a estas prácticas interventivas. La propia naturaleza social de las personas hace emanar una crítica a dichas prácticas a través de sus relatos, expresando que la intervención realizada en términos naturales tiene su efecto en la propia construcción que la persona hace del mundo. A la vez, hay fragmentos en los relatos que se refieren a su experiencia de cuerpo en tercera persona, lenguaje propio de una fisiología reduccionista que entiende el cuerpo como una parte-extra-partes, lo que se enmarca dentro de un lenguaje biomédico occidental que mediatiza y atraviesa las vivencias y sentimientos, penetrando en las narraciones de los protagonistas y haciendo suyo un lenguaje socialmente impuesto de manera tácita -validado históricamente por el colectivo frente a la institución médica-, estableciéndose también una relación de dominación a través de él (6).
Este nos parece otro punto fundamental a considerar en relación a los aportes en términos prácticos de esta investigación, ya que es fundamental el cómo nos posicionamos como Terapeutas Ocupacionales, cómo nos aproximamos a los usuarios y cómo establecemos la relación terapéutica, no desde la exterioridad ni desde una posición de jerarquía sobre el usuario, sino como facilitadores del proceso rescatando siempre la voz de los propio actores. Nosotros como T.O. nos queremos hacer cargo más allá de una intervención en términos naturales, más allá de ver al cuerpo como una máquina que se construye de palancas, torques, y que dan origen a la fuerza y el movimiento basada en las leyes de la física y la biología. Nos queremos hacer cargo del cuerpo como un sujeto en sí mismo, nos queremos hacer cargo de su historia, de cómo éste se ha construido como un hecho social e histórico, no queremos intervenir en términos abstractos, centrando nuestra intervención sólo en la parte afectada o en los aspectos disfuncionales, si no que queremos abrir nuestra mirada y contribuir a la posibilidad de que la disciplina también lo haga, principalmente en el ámbito de intervención en salud física, con el objeto de que este cambio se exprese en un replanteamiento paradigmático en el cómo se valora al sujeto en este ámbito de intervención, y consecuentemente promover un cambio en la práctica disciplinar. Para ello creemos que es significativo que este tipo de investigación y el conocimiento generado a partir de ella sea difundido a nivel de formación de futuros Terapeutas Ocupacionales.

\section{ReferenCias Bibliograficas}

(1) Adamson, G. Concepción de Subjetividad en E.P Riviere. (Fecha de revisión 17, marzo, 2004). Disponible en: http://www.geocoties.com/athens/forum/5396/subjetividad.html

(2) FERRANTE, C., FERREIRA M.: Cuerpo y Habitus: el marco estructural de la experiencia de la discapacidad. (2007)(Fecha revisión: 23. Abril. 2009) 
Disponible en: http://www.mferreira.es/Documentos_nuevo/Publicaciones.htm

(3) KORNBLIT, A. Metodologías cualitativas en ciencias sociales: Modelos y procedimientos de análisis. Buenos Aires: Biblios (2007)

(4) CHIRICO, M. Los relatos de vida. El retorno a lo biográfico. Buenos Aires: Centro Editor de América Latina (1992)

(5) CAPRA, F. El punto crucial: ciencia, sociedad y cultura naciente. Buenos Aires: Troquel (1999)

(6) FERREIRA, M. La construcción social de la discapacidad: habitus, estereotipos y exclusión social. (2008) (Fecha revisión: 23. Abril. 2009) Disponible en: http://dialnet.unirioja.es/servlet/ articulo?codigo $=2518583$

(7) FOUCAULT, M. Vigilar y Castigar. Buenos Aires: Siglo XXI, Argentina: (2006).

(8) BUTLER, J. Cuerpos que importan: Sobre los límites materiales y discursivos del "sexo". Buenos Aires: Paidós (2002) 